\title{
THE LOOMAN-MENCHOFF THEOREM AND SOME SUBHARMONIC FUNCTION ANALOGUES ${ }^{1}$
}

\author{
MAYNARD G. ARSOVE
}

1. Introduction. Starting with the work of Goursat [7] in 1900 there has been considerable interest in the following question: given a continuous complex function $f$ on an open set $\Omega$, how weak can one make the further conditions imposed to guarantee analyticity of $f$ ? In particular, conditions have been sought which restrict the behavior of $f$ on as small as possible a subset of $\Omega$. Theorems of this sort are frequently associated with the names of Looman and Menchoff, who were the first to show $[16$, p. 199] that for $f$ sufficiently well behaved the Cauchy-Riemann equations need only be required to hold almost everywhere.

In the sequel it will be convenient for purposes of comparison to have at our disposal two theorems of Besicovitch [3], and we state them here with the same numbering.

THEOREM 1. Let $f$ be a bounded complex function on $\Omega-E$, where $\Omega$ is an open set and $E$ a subset having zero length. ${ }^{2}$ If $f$ admits a finite derivative on $\Omega-E$, then $f$ can be extended so as to be analytic on $\Omega .^{3}$

THEOREM 2. Let $f$ be a continuous complex function on an open set $\Omega$, and let $E$ be the union of countably many subsets of finite length. If $f$ has a finite derivative on $\Omega-E$, then $f$ is analytic on $\Omega$.

With reference to a point raised by Saks [16, p. 201], Maker has applied Theorem 2 to prove $[8$, p. 267] the following generalization of the Looman-Menchoff theorem.

TheOREM 3. Let $f=u+i v$ be a continuous complex function on an open set $\Omega$, and let $E$ be the union of countably many sets of finite length closed in $\Omega$. If $u$ and $v$ have finite partial Dini derivates on $\Omega-E$ and satisfy the Cauchy-Riemann equations $u_{x}=v_{y}, u_{y}=-v_{x}$ almost everywhere, then $f$ is analytic on $\Omega$.

Presented to the International Congress of Mathematicians, September 6, 1954; received by the editors January 18, 1954, and in revised form, May 12, 1954.

1 The research reported upon here was supported (in part) by the National Science Foundation.

2 Length $=$ Carathéodory linear measure.

3 Actually, the statement of the theorem according to Besicovitch presumes $f$ to be defined on $\Omega$, but the present version follows at once by extending $f$ as in the proof of Theorem 4. 
Our principal aim in the present paper is to extend the LoomanMenchoff theorem to subharmonic and $\delta$-subharmonic functions, and to employ the Looman-Menchoff techniques in determining conditions sufficient for absolute continuity of the mass distributions. Throughout the paper we shall consider only functions defined on an arbitrary open set $\Omega$ in the plane, but it is clear that the theorems involving $\delta$-subharmonic functions extend easily to Euclidean spaces of arbitrary dimension.

To obtain a characterization of continuous subharmonic functions (Theorem 6), we employ the operators of Blaschke and Privaloff, and this suggests the possibility of a corresponding condition for analyticity. Exploitation of this approach by means of techniques due to Meier [9] results in the fact that the Cauchy-Riemann equations in Theorem 3 can be replaced by the expression

$$
\underset{r \rightarrow 0}{\liminf } \frac{1}{r^{2}}\left|\int_{C_{r}(z)} f(\zeta) d \zeta\right|=0,
$$

where $C_{r}(z)$ denotes the positively oriented boundary of the rircle of radius $r$ about $z$. A similar condition in terms of a square of side $r$ has been given by Wolff $[16$, p. 196], but there does not seem to be any intrinsic connection between the methods. ${ }^{4}$

In the majority of the applications of the Looman-Menchoff techniques continuity. plays an essential role in establishing closedness of certain sets (the sets $F_{n}$ in our notation). Nevertheless, it is possible to relinquish continuity under favorable conditions, such as those arising in Theorem 7. This is the case also in the following theorem, which bears the same relationship to Theorem 1 as Maker's theorem does to Theorem 2 .

THEOREM 4. Let $f=u+i v$ be a bounded complex function on $\Omega-E$, where $\Omega$ is an open set and $E$ is the union of countably many sets of zero length closed in $\Omega$. If

$$
\limsup _{\zeta \rightarrow z}\left|\frac{f(\zeta)-f(z)}{\zeta-z}\right|<+\infty \quad \text { for } z \text { on } \Omega-E
$$

and the Cauchy-Riemann equations $u_{x}=v_{y}, u_{y}=-v_{x}$ hold almost everywhere on $\Omega-E$, then $f$ can be extended so as to be analytic on $\Omega$.

A proof of Theorem 4 will be given later in $\$ 4$.

In the $\delta$-subharmonic case the exceptional set $E$ is taken as an

- In dealing with rectangles in situations of this sort, essential use is made of their subdivision properties. 
arbitrary Borel $F_{\sigma}$ set of capacity zero (see [6, Chap. VII]). Since the irregular boundary points of any open set comprise an $F_{\sigma}$ of capacity zero, it seems likely that the theorems (or at least the methods) indicated here may have applications related to the Dirichlet problem. In fact, we are led to conjecture that the LoomanMenchoff techniques should be of value in a wide class of problems involving the approximation of linear operators by difference operators. $^{5}$

2. Subharmonic function theorems of Looman-Menchoff type. That harmonic functions admit a Looman-Menchoff characterization has been shown by Beckenbach [2], under the assumption that the exceptional set $E$ is countable.

We observe that modifications of the sort made by Maker $[8, \mathrm{p}$. 266 ] in the proof of the Looman-Menchoff theorem can also be made in the proof of the theorem of Beckenbach, but it is just as easy to establish a more inclusive condition sufficient for subharmonicity.

THEOREM 5. Let $u$ be a real-valued function having continuous first partial derivatives on an open set $\Omega$, and let $E$ be a Borel $F_{\sigma}$ set of capacity zero. If the first partial Dini derivates of $u_{x}$ and $u_{y}$ are finite on $\Omega-E$ and $\Delta u \equiv u_{x x}+u_{y y} \geqq 0$ holds almost everywhere, then $u$ is subharmonic on $\Omega$.

Like all of the currently known proofs of the Looman-Menchoff theorem and its generalizations, our proof of Theorem 5 leans heavily on the Baire category theorem.

Let $F$ be the set of all points $z(\in \Omega)$ such that $u$ is not subharmonic on any neighborhood of $z$. Clearly $F$ is closed in $\Omega$ and $u$ is subharmonic on $\Omega-F$. Suppose that $F \neq \varnothing$.

For positive integral $n$ let us define $F_{n}$ as the set of all points $z(\in \Omega)$ such that $|h|<1 / n$ ( $h$ being real) implies

$$
\begin{aligned}
\mid u_{x}(z+h)- & u_{x}(z)|+| u_{x}(z+i h)-u_{x}(z) \mid \\
& +\left|u_{y}(z+h)-u_{y}(z)\right|+\left|u_{y}(z+i h)-u_{y}(z)\right| \leqq n|h| .
\end{aligned}
$$

The sets $F_{n}$ are closed in $\Omega$ and in their aggregate cover $\Omega-E$. Further, we have $E=\cup_{n=1}^{\infty} E_{n}$, where each $E_{n}$ is a set of capacity zero closed in $\Omega$. Since the countable family of relatively closed sets $F_{n}$ and $E_{n}$ covers $\Omega$, and a fortiori $F$, the Baire category theorem ensures the existence of a neighborhood $\omega$ such that $F \cap \omega$ is nonempty and lies in one of the covering sets.

The possibility of $F \cap \omega$ being contained in some $E_{n}$ is, however,

5 In particular, these techniques should find application in the theory of partial differential equations. 
ruled out by a theorem of Brelot [4, p. 31] which asserts that a function bounded above on a region and subharmonic outside of a relatively closed subset of capacity zero admits a unique subharmonic extension on the region ${ }^{6}$ (by continuity this extension must in our case coincide with $u$ ). There is thus an index $N$ for which $F \cap \omega \subset F_{N}$.

A proof of the following lemma is given in the monograph of Menchoff $[10$, p. 10]. Let $w$ be a continuous real-valued function on a square $Q=[\alpha, \alpha+s] \times[\beta, \beta+s]$, and let $F(\neq \varnothing)$ be a closed subset of $Q$ and $M$ a constant such that $(x, y) \in F$ and $(x+h, y),(x, y+k) \in Q$ imply

$$
|w(x+h, y)-w(x, y)| \leqq M|h|,|w(x, y+k)-w(x, y)| \leqq M|k| .
$$

If $R=\left[x_{1}, x_{2}\right] \times\left[y_{1}, y_{2}\right]$ is the smallest (possibly degenerate) rectangle having edges parallel to the coordinate axes and containing $F$, then

$$
\begin{aligned}
& \left|\int_{x_{1}}^{x_{2}}\left[w\left(x, y_{2}\right)-w\left(x, y_{1}\right)\right] d x-\int_{F} w_{y} d a\right| \leqq 5 M a(R-F), \\
& \left|\int_{y_{1}}^{y_{2}}\left[w\left(x_{2}, y\right)-w\left(x_{1}, y\right)\right] d y-\int_{F} w_{x} d a\right| \leqq 5 M a(R-F),
\end{aligned}
$$

where $a$ denotes Lebesgue plane measure. ${ }^{8}$

Beckenbach [2] has shown how an argument paralleling that used by Menchoff in the analytic case leads at once to

$$
\int_{\partial \boldsymbol{Q}}(\partial u / \partial \nu) d s=\int_{F \cap Q} \Delta u d a \geqq 0
$$

( $\nu$ denoting the outward normal and $\partial Q$ the positively oriented boundary of $Q$ ) for every square $Q \subset \omega$. It is then obvious from the proof of Lemma 3 of Beckenbach [2] that this makes $u$ subharmonic on $\omega$. The resulting contradiction forces $F=\varnothing$, completing the proof.

Replacing the inequality in Theorem 5 by equality plainly results in harmonicity of $u$ and shows that the exceptional set in the theorem of Beckenbach can be allowed to be an $F_{\sigma}$ of capacity zero.

We recall $[1$, p. 335$]$ that for $u$ an integrable function the Blaschke difference operator $\Delta_{r}^{B}$ is defined by

$$
\Delta_{r}^{B} u(z)=\left(4 / r^{2}\right)\left[\mu_{r} u(z)-u(z)\right],
$$

- Although stated in terms of a closed set by Brelot, it is evident that the set need only be relatively closed.

7 Note that the corresponding lemma in Saks [16, p. 198] and in Beckenbach [2] is unnecessarily restrictive.

8 The Lipschitz conditions on $w$ imply that $w_{x}$ and $w_{y}$ exist almost everywhere and are summable on $F[16$, Chap. V]. 
where $\mu_{r} u(z)$ is the integral mean of $u$ over the circumference of the circle of radius $r$ about $z$. Denoting by $\bar{\Delta}^{B} u(z)$ and $\Delta^{B} u(z)$, respectively, the limit superior and limit inferior of $\Delta_{r}^{B} u(z)$ as $r \rightarrow 0$, we obtain the upper and lower Blaschke operators, $\bar{\Delta}^{B}$ and $\Delta^{B}$. Use of the areal mean $\alpha_{r} u(z)$ in place of the circumferential mean $\mu_{r} u(z)$, and the factor $8 / r^{2}$ in place of $4 / r^{2}$, serves to define the corresponding Privaloff operators $\Delta_{r}^{P}, \bar{\Delta}^{P}$, and $\Delta^{P}$ analogously.

As we proceed to show, a Looman-Menchoff criterion for continuous functions to be subharmonic can be given in terms of the operators of Blaschke and Privaloff. ${ }^{9}$

THEOREM 6. Let $u$ be a continuous real-valued function on an open set $\Omega$. Then for $u$ to be subharmonic on $\Omega$ it is necessary and sufficient that $\Delta^{B} u>-\infty$ hold except perhaps on a Borel $F_{\sigma}$ set of capacity zero and that $\Delta^{B} u \geqq 0$ hold almost everywhere.

Proof. The necessity is obvious. For the sufficiency we take $F$ as the set of all points $z(\in \Omega)$ such that $u$ is not subharmonic on any neighborhood of $z$, and define $F_{n}$ as the set of all points $z(\in \Omega)$ such that $0<r<1 / n$ implies $\Delta_{r}^{B} u(z) \geqq-n$. These sets are closed in $\Omega$ by virtue of the continuity of $\Delta_{r}^{B} u$, so that if $F$ is not empty, we conclude exactly as in the proof of Theorem 5 that $F_{N} \supset F \cap \omega \neq \varnothing$ for some index $N$ and some neighborhood $\omega$.

Now, from $\Delta^{B} u \geqq-N$ on $\omega$, it follows ${ }^{10}$ that the function defined by $u(x, y)+N x^{2} / 2$ is subharmonic on $\omega$, and there results $\Delta_{r}^{B} u \geqq-N$ on the neighborhood obtained by shrinking $\omega$ radially through a distance $r$. It is evident also that the areal mean operator $\alpha_{\rho}$ commutes with the Blaschke difference operator to yield $\Delta_{r}^{B} \alpha_{\rho} u=\alpha_{\rho} \Delta_{r}^{B} u$. Thus, taking advantage of the fact that the functions $\Delta_{r}^{B} u$ are bounded below, we can apply Fatou's lemma to infer

$$
\underline{\Delta}^{B} \alpha_{\rho} u \geqq \alpha_{\rho} \Delta^{B} u \geqq 0 .
$$

This implies $\left[13\right.$, p. 14] that the continuous functions $\alpha_{\rho} u$ are subharmonic. Since they converge uniformly to $u$ on compact subsets, $u$ must be subharmonic on $\omega$, contradicting our assumption of $F \neq \varnothing$.

Observe that the hypothesis of continuity on $u$ cannot be supplanted by even such a plausible condition as $\lim \sup _{\zeta \rightarrow z} u(\zeta)=u(z)$ for all $z \in \Omega$. This follows from the fact that there exist nonempty perfect sets of capacity zero [11, p. 153] (the function $u$ defined as 1 on such a set and 0 elsewhere is not subharmonic).

- We carry out the details only for the case of the Blaschke operator, but the Privaloff operator behaves in the same way.

${ }^{10}$ See, for example, the proof of Theorem 19 of [1]. 
It is of interest to compare Theorem 6 with the following theorem of Privaloff [12]: a necessary and sufficient condition for a continuous real-valued function $u$ to be subharmonic on an open set $\Omega$ is that $\bar{\Delta}^{P} u>-\infty$ hold except possibly on a closed set of capacity zero and $\bar{\Delta}^{P} u \geqq 0$ hold almost everywhere. To allow the exceptional set to be an $F_{\sigma}$, we have thus had to pay only the rather reasonable price of replacing the upper by the lower operator.

An immediate consequence of Theorem 6 is

Corollary 6.1. A real-valued function $h$ on an open set $\Omega$ is harmonic if and only if $h$ is continuous, lim $\sup _{r \rightarrow 0}\left|\Delta_{r}^{B} h\right|$ is finite except perhaps on a Borel $F_{\sigma}$ set of capacity zero, and $\Delta^{B} h=0$ almost everywhere.

For $\delta$-subharmonic generalizations of Theorems 5 and 6 we have recourse to an elementary argument of the kind used in proving Theorem 19 of [1]. It follows that if, instead of requiring the operators appearing in these theorems to be non-negative almost everywhere, we require them to be locally essentially bounded below, then the function $u$ is $\delta$-subharmonic on $\Omega$.

3. Absolute continuity of the mass distributions. Aside from yielding conditions for subharmonicity and $\delta$-subharmonicity, the Looman-Menchoff techniques can be applied to the problem of determining when the mass distribution for a subharmonic or $\delta$-subharmonic function is absolutely continuous (that is, is given as the Lebesgue integral of a summable density function).

In developing some sufficient conditions of this sort, we shall have use for two preliminary lemmas. The first of these lemmas is a special case of Theorem I of Rudin [15], but we present a brief independent proof based on Rosenbloom's characterization of the mass distribution for a potential [14].11 It will be convenient to have on hand the following notational conventions: (1) for $N$ any real number, $\lambda_{N}$ will signify the function defined on the plane by

$$
\lambda_{N}(x, y)=N x^{2} / 2 ;
$$

(2) for $\Omega$ an open set and $r$ a positive number, $\Omega_{r}$ will signify the set of all points of $\Omega$ whose distance from the boundary exceeds $r$.

LEMMA 1. Let $w$ be a real-valued function continuous on an open set $\Omega$. If $\Delta^{B} w$ is bounded above and $\bar{\Delta}^{B} w$ is bounded below, then $w$ is $\delta$-subharmonic and its mass distribution is absolutely continuous.

Proof. Choosing $N$ such that $\underline{\Delta}^{B} w \leqq N$ and $\bar{\Delta}^{B} w \geqq-N$, we see

${ }^{11}$ See also Theorem 13 of [1]. 
that $\lambda_{N}-w$ and $\lambda_{N}+w$ are subharmonic. There results the boundedness condition

$$
-N \leqq \Delta_{r}^{B} w \leqq N
$$

on $\Omega_{r}$. Since $w$ is $\delta$-subharmonic, a lemma of Rudin [15, p. 280] assures us that $\Delta^{B} w$ exists almost everywhere and is summable on compact subsets of $\Omega$. Hence, if $m$ is the mass distribution for $w$, the theorem of Rosenbloom mentioned above yields

$$
-m(e)=\lim _{r \rightarrow 0} \frac{1}{2 \pi} \int_{0} \Delta_{r}^{B} w d a=\frac{1}{2 \pi} \int_{0}^{B} \Delta^{B} w d a
$$

for all bounded Borel sets $e$ with closure in $\Omega$ and having no mass on their boundaries. The lemma is thus established.

The second lemma is readily reduced to the preceding one.

LemMA 2. Let $\Omega$ be an open set, $F$ a subset closed in $\Omega$, and $U$ the potential of a negative mass distribution $m$ on $F$. If for some constant $N$ and all sufficiently small $r(>0)$ the inequality

$$
\Delta_{r}^{B} U \leqq N
$$

holds on $F \cap \Omega_{r}$, then $U$ is continuous on $\Omega$ and $m$ is absolutely continuous.

Proof. Taking $z$ and $z_{0}$ as points of $F$, it being assumed further that $z_{0}$ is a limit point, we have for small $r$

$$
\begin{aligned}
\left|U(z)-U\left(z_{0}\right)\right| \leqq & \left|U(z)-\mu_{r} U(z)\right|+\left|\mu_{r} U(z)-\mu_{r} U\left(z_{0}\right)\right| \\
& +\left|\mu_{r} U\left(z_{0}\right)-U\left(z_{0}\right)\right| \\
\leqq & \left|\mu_{r} U(z)-\mu_{r} U\left(z_{0}\right)\right|+N r^{2} / 2 \underset{z \rightarrow z_{0}}{\longrightarrow} N r^{2} / 2
\end{aligned}
$$

by virtue of the continuity of $\mu_{r} U$. Hence, $U$ is continuous as a function on $F$, and it follows from the work of Evans [5, p. 238] that $U$ must be continuous on $\Omega$. An application of Lemma 1 completes the proof.

With this information at our disposal we are in a position to prove a theorem related to Theorem II of Rudin [15]. The distinction here is similar to that between Theorem 6 and the theorem of Privaloff noted in connection with it: we replace the lower by the upper operator but allow the exceptional set to be an $F_{\sigma}$. However, in the present case we receive the unexpected bonus of not having to require continuity. 
THEOREM 7. Let $\Omega$ be an open set and $u$ a subharmonic function on $\Omega$ having the property that the set $\{z: u(z)=-\infty\}$ supports none of the mass of $u$. If $\bar{\Delta}^{B} u<+\infty$, except perhaps on a Borel $F_{\sigma}$ set of capacity zero, then the mass distribution for $u$ is absolutely continuous and there exists a subset $E$ of capacity zero closed in $\Omega$ such that $\bar{\Delta}^{B} u<+\infty$ on $\Omega-E$.

Proof. We take $F$ as the set of all points $z(\in \Omega)$ such that on no neighborhood $\nu$ of $z$ does $u$ satisfy the following conditions: (i) the mass distribution $m$ for $u$ is absolutely continuous on $\nu$, and (ii) for some subset $A_{\nu}$ of capacity zero closed in $\nu$ we have $\bar{\Delta}^{B} u<+\infty$ on $\nu-A_{\nu}$. It is obvious that $F$ is closed in $\Omega$. Moreover, $u$ possesses properties (i) and (ii) on the open set $\nu=\Omega-F$, as is easily seen by paracompactness considerations. Let us suppose that $F$ is nonempty.

For $n=1,2, \cdots$ we denote by $F_{n}$ the set of all points $z(\in \Omega)$ such that $0<r<1 / n$ implies $\triangle_{r}^{B} u(z) \leqq n$. Invoking the continuity of $\mu_{r} u$ and the fact that $\lim \sup _{z \rightarrow z_{0}} u(z)=u\left(z_{0}\right)$, we find that

$$
\Delta_{r}^{B} u\left(z_{0}\right)=\liminf _{z \rightarrow z_{0}} \Delta_{r}^{B} u(z) \leqq \liminf _{z\left(\in F_{n}\right) \rightarrow z_{0}} \Delta_{r}^{B} u(z) \leqq n
$$

for $0<r<1 / n$ and $z_{0}$ a limit point of $F_{n}$. The sets $F_{n}$ are thus closed in $\Omega$.

It is evident that $\Omega$ is covered by the sets $F_{n}$ and $E_{n}(n=1,2, \cdots)$, the latter being suitably chosen sets of capacity zero closed in $\Omega$. Hence, there exists a neighborhood $\omega(C \Omega)$ such that $F \cap \omega$ is nonempty and lies in one of the covering sets. This cannot occur, however, for any $E_{n}$, in view of our hypothesis prohibiting sets of capacity zero from supporting any of the mass of $u$. It follows that $F \cap \omega \subset F_{N}$ for some index $N$, so that

$$
\Delta_{r}^{B} u(z) \leqq N
$$

for $z$ on $F \cap \omega$ and $0<r<1 / N$.

We observe that this inequality remains valid for the potential $U$ obtained by restricting $m$ to $F \cap \omega$, inasmuch as $u$ is the sum of $U$ and a subharmonic function. Lemma 2 now applies to $U$, establishing absolute continuity of $m$ on $F \cap \omega$, and the resulting contradiction shows that $F$ is empty.

Combining this result with Theorem 6 gives rise to a direct extension to the $\delta$-subharmonic case.

COROLlary 7.1. Let $w$ be a real-valued function continuous on an open set $\Omega$. If $\bar{\Delta}^{B} w$ and $\underline{\Delta}^{B} w$ are finite, except perhaps on a Borel $F_{\sigma}$ set 
of capacity zero, and if $\Delta^{B} w$ is locally essentially bounded below, then $w$ can be represented as the difference of two continuous subharmonic functions having absolutely continuous mass distributions.

The proof is based on consideration of the function $w-\lambda_{c}$ on a neighborhood over which $c$ is an essential lower bound for $\Delta^{B} w$. Theorem 6 shows $w-\lambda_{c}$ to be subharmonic, and Theorem 7 shows its mass distribution to be absolutely continuous.

We then make use of the following generalization of Poisson's equation, given by Rudin $[15,3.6]$ : if the mass distribution for a $\delta$-subharmonic function $w$ is absolutely continuous and has density $\rho$, then $\Delta^{B} w=-2 \pi \rho$ almost everywhere. Since the potential of a mass distribution defined by a bounded density function is continuous, it is apparent by splitting $\Delta^{B} w / 2 \pi$ into its positive and negative parts that the function $w$ in Corollary 7.1 can be represented as the difference of two continuous subharmonic functions having absolutely continuous mass distributions.

4. The analytic case. Proof of Theorem 4. Let us first of all verify the assertion made in $\$ 1$ that Theorem 3 remains in force when the Cauchy-Riemann equations are replaced by the equation

$$
\liminf _{r \rightarrow 0} \frac{1}{r^{2}}\left|\int_{C_{r}(z)} f(\zeta) d \zeta\right|=0 .
$$

We take $F$ as the set of all points $z(\in \Omega)$ such that $f$ is not analytic on any neighborhood of $z$, and for positive integral $n$ define $F_{n}$ as the set of all points $z(\in \Omega$ ) such that $|h|<1 / n$ ( $h$ real) implies

$$
|f(z+h)-f(z)|+|f(z+i h)-f(z)| \leqq n|h| \text {. }
$$

Under the assumption that $F$ is nonempty the application of Besicovitch's theorem employed by Maker [8, p. 267] serves to establish the existence of a neighborhood $\omega$ and an index $N$ such that $F_{N} \supset F$ $\cap \omega \neq \varnothing$.

From a result of Meier $[9$, p. 186] it is evident that $\omega$ can be chosen in such a way that $f$ satisfies a Lipschitz condition

$$
|f(\zeta)-f(z)| \leqq M|\zeta-z|
$$

for $\zeta, z$ on $\omega$. Also, the continuity of $u$ implies that the areal mean function $\alpha_{r} u$ is continuously differentiable and in fact $[13, \mathrm{p} .11]$ has the partial derivatives

$$
\frac{\partial \alpha_{r} u(z)}{\partial x}=\frac{1}{\pi r} \int_{0}^{2 \pi} u\left(z+r e^{i \theta}\right) \cos \theta d \theta
$$




$$
\frac{\partial \alpha_{r} u(z)}{\partial y}=\frac{1}{\pi r} \int_{0}^{2 \pi} u\left(z+r e^{i \theta}\right) \sin \theta d \theta .
$$

Since the Lipschitz condition guarantees the existence almost everywhere of $u_{x}, u_{y}$ [16, Chap. V] and justifies differentiating under the integral sign, we have access to the further identities

$$
\left(\alpha_{r} u\right)_{x}=\alpha_{r}\left(u_{x}\right) \text { and }\left(\alpha_{r} u\right)_{y}=\alpha_{r}\left(u_{y}\right) .
$$

Similar expressions hold for $v$, and there results

$$
\frac{1}{\pi r^{2}} \int_{C_{r}(z)} f(\zeta) d \zeta=i\left[\alpha_{r} u_{x}(z)-\alpha_{r} v_{y}(z)\right]-\left[\alpha_{r} u_{\nu}(z)+\alpha_{r} v_{x}(z)\right] .
$$

In the limit as $r \rightarrow 0$ this yields $u_{x}=v_{y}$ and $u_{y}=-v_{x}$ almost everywhere on $\omega$. Hence, from Theorem 3 (or the observation that the analytic functions $\alpha_{r} u+i \alpha_{r} v$ converge uniformly on compact subsets to $u+i v$ ) it is apparent that $f$ is analytic on $\omega$, so that $F=\varnothing$.

This completes the proof of our assertion, and we turn to the demonstration of Theorem 4.

Here the function $f$ is given only on $\Omega-E$, but we immediately extend it to all of $\Omega$ in the following way. With each point $z$ of $E$ we associate arbitrarily a sequence $\left\{\zeta_{k}(z)\right\}$ of points of $\Omega-E$ such that $\lim _{k \rightarrow \infty} \zeta_{k}(z)=z$ and $\left\{f\left[\zeta_{k}(z)\right]\right\}$ is convergent; this is clearly possible since $f$ is bounded. Then $f(z)$ is specified as the limit of $\left\{f\left[\zeta_{k}(z)\right]\right\}$.

Having thus defined $f$ on $\Omega$, we let $F$ be the set (closed in $\Omega$ ) consisting of all points $z(\in \Omega)$ for which $f$ is not analytic on any neighborhood of $z$. As in the preceding proofs we assume that $F$ is not empty and from this trace a contradiction.

For positive integral $n, F_{n}$ will denote the set of all points $z(\in \Omega)$ such that $|\zeta-z|<1 / n$ implies

$$
|f(\zeta)-f(z)| \leqq n|\zeta-z| \cdot
$$

The following direct argument shows that each $F_{n}$ is closed in $\Omega$. Take $z_{0}$ as a limit point of $F_{n}, \zeta$ as a point of $\Omega$ such that $\left|\zeta-z_{0}\right|$ $<1 / n, \epsilon$ as the positive number $1 / n-\left|\zeta-z_{0}\right|$, and $\left\{z_{k}\right\}$ as any sequence of points of $F_{n}$ converging to $z_{0}$ and satisfying $\left|z_{k}-z_{0}\right|<\epsilon$. Since $\left|\zeta-z_{k}\right|<1 / n$ and $\left|z_{k}-z_{0}\right|<1 / n$, we find

$$
\begin{aligned}
\left|f(\zeta)-f\left(z_{0}\right)\right| & \leqq\left|f(\zeta)-f\left(z_{k}\right)\right|+\left|f\left(z_{0}\right)-f\left(z_{k}\right)\right| \\
& \leqq n\left(\left|\zeta-z_{k}\right|+\left|z_{0}-z_{k}\right|\right) \underset{k \rightarrow \infty}{\longrightarrow} n\left|\zeta-z_{0}\right| .
\end{aligned}
$$

Moreover, the method used in extending $f$ ensures that the sets $F_{n}$ cover $\Omega-E$. That is, the inequality $\left(^{*}\right)$ holds at each point $z$ of 
$\Omega-E$ for some corresponding $n$ and all $\zeta$ on $\Omega-E$ which satisfy $|\zeta-z|<1 / n$; by passing to the limit through appropriate points of $\Omega-E$, it therefore holds for all $\zeta$ on $\Omega$ satisfying $|\zeta-z|<1 / n$.

Applying Theorem 1 in conjunction with the Baire category theorem, we obtain a neighborhood $\omega$ centered at some point $a$ of $F$ and an index $N$ such that $F_{N} \supset F \cap \omega$. This forces $f$ (analytic on $\omega-F$ ) to be continuous on $\omega$. Shrinking $\omega$ if necessary, we shall suppose that its radius is less than $1 / N$. Once again we refer to the work of Meier $[9$, p. 186] for the fact (easily established by means of the Cauchy integral formula) that the Lipschitz condition

$$
|f(\zeta)-f(z)| \leqq 2 N|\zeta-z|
$$

then holds for all $\zeta, z$ on the neighborhood $\omega^{\prime}$ about $a$ having radius half that of $\omega$.

With the Lipschitz condition at our disposal it becomes profitable to introduce the areal mean functions $\alpha_{r} u$ and $\alpha_{r} v$. That is, we are assured that the integrand in the identity

$$
\begin{aligned}
\frac{\alpha_{r} u(z+h)-\alpha_{r} u(z)}{h}-\frac{\alpha_{r} v(z+i h)-\alpha_{r} v(z)}{h} \\
=\frac{1}{\pi r^{2}} \int_{0}^{r} \int_{0}^{2 \pi}\left[\frac{u\left(z+h+\rho e^{i \theta}\right)-u\left(z+\rho e^{i \theta}\right)}{h}\right. \\
\left.-\frac{v\left(z+i h+\rho e^{i \theta}\right)-v\left(z+\rho e^{i \theta}\right)}{h}\right] \rho d \theta d \rho
\end{aligned}
$$

is bounded for $h(\neq 0)$ real and numerically small. Since, as $h \rightarrow 0$, the integrand tends to 0 almost everywhere (through values for which the argument belongs to $\Omega-E$, and therefore, by continuity, through all values), there results

$$
\partial \alpha_{r} u / \partial x=\partial \alpha_{r} v / \partial y .
$$

Similarly,

$$
\partial \alpha_{r} u / \partial y=-\partial \alpha_{r} v / \partial x .
$$

The functions $f_{r}=\alpha_{r} u+i \alpha_{r} v$, which have just been shown to be analytic, converge uniformly on compact subsets of $\omega^{\prime}$ to $f$, so that $f$ must be analytic on $\omega^{\prime}$. This contradiction proves that $f$, as extended, is analytic on $\Omega$.

It should be remarked that the proof given here can be somewhat shortened by invoking the classical Looman-Menchoff theorem for $f$ on $\omega$. The development actually adopted is, however, substantially more elementary. 
5. Added in proof. The author is indebted to Professor J. Ridder for calling attention to the theorem enunciated in footnote 31 of his paper Harmonische, subharmonische und analytische Funktionen [Annali Scuola Normale Superiore, Pisa (2) vol. 9 (1940) pp. 277-287].

This theorem implies that the exceptional set $E$ in Theorem 4 can actually be taken as the union of countably many sets of zero length, not necessarily closed in $\Omega$. However, the derivation outlined by Ridder is based on fairly deep results of Saks and Zygmund, Haslam Jones, and Morera-Rademacher, whereas Theorem 4 follows in an elementary way from Besicovitch's Theorem 1. On the other hand, it is inherent in the nature of the Looman-Menchoff argument that the exceptional set must be an $F_{\sigma}$ for this argument to be applicable.

\section{BIBLIOGRAPHY}

1. M. G. Arsove, Functions representable as differences of subharmonic functions, Trans. Amer. Math. Soc. vol. 75 (1953) pp. 327-365.

2. E. F. Beckenbach, Concerning the definition of harmonic functions, Bull. Amer. Math. Soc. vol. 51 (1945) pp. 240-245.

3. A. S. Besicovitch, On sufficient conditions for a function to be analytic, and on behavior of analytic functions in the neighborhood of non-isolated singular points, Proc. London Math. Soc. vol. 32 (1931) pp. 1-9.

4. M. Brelot, Étude des fonctions sousharmoniques au voisinage d'un point, Actualités Scientifiques et Industrielles, vol. 139, 1934, pp. 1-55.

5. G. C. Evans, On potentials of positive mass (Part I), Trans. Amer. Math. Soc. vol. 37 (1935) pp. 226-253.

6. O. Frostman, Potentiel d'équilibre et capacité des ensembles avec quelques applications a la théorie des fonctions, Lunds Univ. Math. Seminar vol. 3 (1935) pp. 1-115.

7. E. Goursat, Sur la définition générale des fonctions analytiques d'après Cauchy, Trans. Amer. Math. Soc. vol. 1 (1900) pp. 14-16.

8. P. T. Maker, Conditions on $u(x, y)$ and $v(x, y)$ necessary and sufficient for the regularity of $u+i v$, Trans. Amer. Math. Soc. vol. 45 (1939) pp. 265-275.

9. K. Meier, Zum Satz von Looman-Menchoff, Comment. Math. Helv. vol. 25 (1951) pp. 181-195.

10. D. Menchoff, Les conditions de monogénéité, Actualités Scientifiques et Industrielles, vol. 329, 1936, pp. 1-52.

11. R. Nevanlinna, Eindeutige analytische Funktionen, Berlin, 1936.

12. I. I. Privaloff, Sur la définition d'une fonction harmonique, Doklady Akad. Nauk SSSR. N.S. vol. 31 (1941) pp. 102-103.

13. T. Rad6, Subharmonic functions, Berlin, 1937.

14. P. C. Rosenbloom, Mass distributions and their potentials, Proceedings of the 11th Scandinavian Mathematical Congress, Trondheim, Norway, 1949, pp. 130-138.

15. W. Rudin, Integral representation of continuous functions, Trans. Amer. Math. Soc. vol. 68 (1950) pp. 278-286.

16. S. Saks, Theory of the integral, 2d rev. ed., Warsaw, 1937.

UNIVERSITY OF WASHINGTON 